\title{
Drought drove forest decline and dune building in eastern Upper Michigan USA as the upper Great Lakes became closed basins
}

Optical ages, supporting data and site details are found in Tables DR1 and DR2.

Optically stimulated luminescence (OSL) methodology

Samples were collected between the surface and $2 \mathrm{~m}$ depth by driving $20 \mathrm{~cm} \times 7.5 \mathrm{~cm}$ sections of aluminum tubing into vertical walls of soil pits or exposures. Where possible, samples were taken from the $\mathrm{C}$ horizon in order to minimize effects of pedoturbation and/or pedogenesis. In many sites, samples were taken directly in eolian sand containing sedimentary structures. Eolian silt cap samples ( 6 of 8 ) were taken from $<1 \mathrm{~m}$ depth due to the thin (on average $<50 \mathrm{~cm}$ thickness) nature of the silt cap (Schaetzl and Loope, 2008). Additional sediment samples were taken within $30 \mathrm{~cm}$ of the sampling tube for determination of water content and for chemical analysis. In-situ water content was measured by weight loss upon drying at $60^{\circ} \mathrm{C}$ for 24 hours. Elemental concentrations (U, Th, K, Rb) for the calculation of the dose rate (Aitken, 1998) were determined by ICP-MS, ICP-AES, and XRFS at Chemex Laboratories, Sparks, Nevada and by high-resolution gamma spectrometry (Preusser and Kasper, 2001; Lavi et al., 2004) at the Luminescence Geochronology Lab at the University of Nebraska-Lincoln. The cosmic ray contribution to the dose rate was calculated from Prescott and Hutton (1994). The dose rate and associated errors were calculated using Aitken and Alldred (1972), Aitken (1976, 1985), Adamiec and Aitken (1998), Aitken (1998), and Brennan (2003).

Samples were processed at the Luminescence Geochronology Lab in the Department of Earth and Atmospheric Sciences at the University of Nebraska-Lincoln. Samples were opened under dim amber light and the outer $\sim 5 \mathrm{~cm}$ of sediment was removed from the ends of the tube to avoid contamination by bleached grains. The remaining sample was wet sieved to isolate the 90 $150 \mu \mathrm{m}$ fraction, treated with $1 \mathrm{~N} \mathrm{HCl}$ to remove carbonates and a citrate-dithionite-bicarbonate (CBD) solution to remove iron oxide staining. Quartz and feldspar was separated from heavy minerals by flotation in sodium polytungstate $\left(\rho=2.7 \mathrm{~g} / \mathrm{cm}^{3}\right)$. Quartz grains were etched and feldspars removed by $48 \% \mathrm{HF}$ ( 75 minutes) and $47 \% \mathrm{HCl}$ (30 minutes) treatments. Samples were then resieved to remove the $<90 \mu \mathrm{m}$ fraction. Quartz grains were mounted on aluminum disks with a $5 \mathrm{~mm}$ mask ( 1000 grains per disk) using silicon spray. Infrared stimulation was used to check for feldspar contamination. Optical measurements were performed on both Risø TL/OSL-DA$15 \mathrm{~B} / \mathrm{C}$ and TL/OSL-DA-20 readers.

Equivalent doses were determined using the single aliquot regenerative (SAR) protocol (Murray and Wintle, 2003). Four regenerative doses were used to bracket the natural signal, with the fourth dose being a repeat of the first regenerative dose. Regenerative growth curves were fit with quadratic, saturating exponential, or saturating exponential with linear component equations to estimate the equivalent dose. Test doses ( 2 Gy) were applied after measurement of the natural and regenerative signal to monitor changes in sensitivity. Preheat plateau and dose recovery tests were performed based on Murray and Wintle (2003). Based on these tests, a $240^{\circ} \mathrm{C}, 10$ second 
preheat and $220^{\circ} \mathrm{C}$ cutheat was used for eolian sand samples and a $220^{\circ} \mathrm{C}, 10$ second preheat and $160^{\circ} \mathrm{C}$ cutheat was used for eolian silt samples. Dose recovery tests were run on 36 samples and returned an average of $0.99 \pm 0.05$ (measured dose / given dose; 1 sigma error), suggesting the SAR protocol is valid for these samples (Wintle and Murray, 2006). Optical measurements were made at $125^{\circ} \mathrm{C}$. Representative OSL data from this study is plotted in Figure DR1.

Optical ages were calculated using the central age model of Galbraith et al. (1999), based on protocols from Bailey and Arnold (2006). In $\mathrm{D}_{\mathrm{e}}$ analysis, individual aliquots were accepted or rejected based on recycling ratios (above $\pm 10 \%$ ) (Murray and Olley, 2002), test dose and/or $\mathrm{D}_{\mathrm{e}}$ error $(>10 \%)$, anomalous shine-down curves indicating contribution from non-quartz sources or intermediate/slow components of the OSL signal. $\mathrm{D}_{\mathrm{e}}$ frequency distributions (Olley et al., 1998, 1999), cumulative probability plots, radial plots (Galbraith, 1988, 2010), plots of standardized $D_{e}$ vs. signal intensity (Colls et al., 2001), and $\mathrm{D}_{\mathrm{e}}(\mathrm{t})$ plots (Bailey et al., 2003) were analyzed in order to detect potential partial bleaching. Ages are reported in thousands of years $(\mathrm{ka})$ before 2007.

\section{Spatial distribution, elevation and optical ages}

To further document the spatial distribution of optical ages, we replaced sample numbers of Figure 1 of the main text with ages (Fig. DR2). Additionally, we plotted age vs. elevation (Fig. DR3) to demonstrate the lack of relationship of dune and silt cap age with elevation.

We contend that the central tendency (mean, median) of the large number of dune ages from this study ( $\mathrm{n}=57$ ), while showing variability, represents the best estimate of the timing of eolian activity in eastern Upper Michigan (10-8 ka).

Additional detailed work is needed to answer questions regarding the differences between optical ages from Arbogast et al. (2002) and this study. The differences in ages are hard to explain, given that mean age from both studies overlap at $2 \sigma$. Both studies used the same methodology (e.g., single aliquot regenerative protocol) but consistent older ages were produced from this study. Analytical errors associated with determination of chemistry may explain the variability and differences in ages between studies, but more work is needed (e.g., high-resolution age and dose rate control on individual dunes) before this can be resolved. 
Table DR1. Optically stimulated luminescence (OSL) ages and supporting data

Eolian dune ages

\begin{tabular}{|c|c|c|c|c|c|c|c|c|c|c|c|c|}
\hline Sample & $\begin{array}{c}\text { Site } \\
\#\end{array}$ & $\begin{array}{c}\text { Depth } \\
\text { (m) }\end{array}$ & $\begin{array}{l}\mathrm{H}_{2} \mathrm{O}^{*} \\
(\mathrm{wt} \%)\end{array}$ & $\begin{array}{l}\mathrm{K}_{2} \mathrm{O} \\
(\mathrm{wt} \%)\end{array}$ & $\begin{array}{c}\mathrm{U} \\
(\mathrm{ppm})\end{array}$ & $\begin{array}{c}\text { Th } \\
(\mathrm{ppm})\end{array}$ & $\begin{array}{l}D_{\text {cosmic }^{\dagger}} \\
\text { (Gy/ka) }\end{array}$ & $\begin{array}{c}D_{\text {total }} \\
(\mathrm{Gy} / \mathrm{ka})\end{array}$ & $\begin{array}{l}D_{\mathrm{e}}^{\S} \\
(\mathrm{Gy}) \\
\end{array}$ & $\begin{array}{rr}\mathrm{Age}^{\#} & \mathrm{~A} \\
(\mathrm{ka}) & \\
\end{array}$ & $\begin{array}{l}\text { Aliquots** } \\
\text { (n) }\end{array}$ & $\begin{array}{l}\mathrm{OD}^{\dagger \dagger} \\
(\%)\end{array}$ \\
\hline UNL1212 & 1 & 1.0 & 3.3 & 2.31 & 0.6 & 2.3 & 0.19 & $2.31 \pm 0.09$ & $20.31 \pm 0.74$ & $8.8 \pm 0.6$ & 29 & 17 \\
\hline UNL1208 & 2 & 1.0 & 3.5 & 2.45 & 0.5 & 2.2 & 0.19 & $2.39 \pm 0.09$ & $13.28 \pm 0.45$ & $5.6 \pm 0.3$ & 29 & 16 \\
\hline UNL1213 & 3 & 1.0 & 3.8 & 1.55 & 0.5 & 2.1 & 0.19 & $1.67 \pm 0.07$ & $16.92 \pm 0.55$ & $10.2 \pm 0.6$ & 31 & 17 \\
\hline UNL1207 & 4 & 1.0 & 3.0 & 1.08 & 0.4 & 1.5 & 0.19 & $1.24 \pm 0.05$ & $11.75 \pm 0.51$ & $9.5 \pm 0.6$ & 29 & 21 \\
\hline UNL1211 & 5 & 1.0 & 2.7 & 1.22 & 0.5 & 2.3 & 0.19 & $1.44 \pm 0.06$ & $13.65 \pm 0.76$ & $9.5 \pm 0.7$ & 27 & 25 \\
\hline UNL881 & 6 & 1.0 & 3.0 & 1.16 & 0.4 & 1.7 & 0.19 & $1.32 \pm 0.05$ & $12.79 \pm 0.52$ & $9.7 \pm 0.6$ & 49 & 23 \\
\hline UNL892 & 7 & 1.0 & 3.4 & 1.88 & 0.5 & 1.6 & 0.19 & $1.90 \pm 0.08$ & $15.60 \pm 0.54$ & $8.2 \pm 0.5$ & 45 & 21 \\
\hline UNL1688 & 8 & 1.0 & 7.7 & 2.77 & 0.6 & 2.5 & 0.19 & $2.56 \pm 0.10$ & $24.19 \pm 1.07$ & $9.4 \pm 0.6$ & 23 & 21 \\
\hline UNL821 & 9 & 1.0 & 4.1 & 1.69 & 0.7 & 3.3 & 0.19 & $1.90 \pm 0.08$ & $16.27 \pm 0.72$ & $8.5 \pm 0.6$ & 28 & 16 \\
\hline UNL1411 & 10 & 1.0 & 5.0 & 3.40 & 0.6 & 2.3 & 0.19 & $3.15 \pm 0.12$ & $30.07 \pm 0.83$ & $9.6 \pm 0.6$ & 20 & 11 \\
\hline UNL2514 & 14 & 1.0 & 5.0 & 2.17 & 1.17 & 2.71 & 0.19 & $2.26 \pm 0.16$ & $19.00 \pm 0.90$ & $8.4 \pm 0.8$ & 20 & 24 \\
\hline UNL879 & 15 & 1.0 & 6.6 & 2.57 & 0.5 & 1.9 & 0.19 & $2.38 \pm 0.10$ & $20.44 \pm 0.66$ & $8.6 \pm 0.5$ & 64 & 24 \\
\hline UNL2510 & 16 & 1.0 & 5.0 & 1.96 & 1.23 & 2.32 & 0.19 & $2.09 \pm 0.14$ & $17.20 \pm 0.90$ & $8.2 \pm 0.8$ & 22 & 23 \\
\hline UNL894 & 17 & 1.0 & 4.3 & 1.83 & 0.6 & 2.0 & 0.19 & $1.89 \pm 0.08$ & $13.69 \pm 0.81$ & $7.2 \pm 0.6$ & 35 & 34 \\
\hline UNL2509 & 18 & 1.3 & 5.0 & 2.36 & 1.95 & 2.00 & 0.19 & $2.52 \pm 0.18$ & $16.60 \pm 0.90$ & $6.6 \pm 0.6$ & 20 & 26 \\
\hline UNL883 & 19 & 1.0 & 5.6 & 1.81 & 0.5 & 1.8 & 0.19 & $1.82 \pm 0.08$ & $16.38 \pm 0.51$ & $9.0 \pm 0.5$ & 35 & 19 \\
\hline UNL2511 & 20 & 0.8 & 5.0 & 1.51 & 1.80 & 1.96 & 0.19 & $1.87 \pm 0.12$ & $14.80 \pm 0.50$ & $7.9 \pm 0.7$ & 20 & 12 \\
\hline UNL2512 & 21 & 0.8 & 5.0 & 1.42 & 1.54 & 1.76 & 0.19 & $1.73 \pm 0.11$ & $11.70 \pm 0.50$ & $6.7 \pm 0.6$ & 22 & 17 \\
\hline UNL895 & 22 & 1.0 & 4.3 & 1.96 & 0.5 & 1.6 & 0.19 & $1.96 \pm 0.08$ & $18.65 \pm 0.65$ & $9.5 \pm 0.6$ & 35 & 20 \\
\hline UNL1692 & 26 & 1.0 & 5.0 & 1.35 & 0.5 & 1.6 & 0.19 & $1.45 \pm 0.06$ & $15.30 \pm 0.53$ & $10.5 \pm 0.7$ & 23 & 15 \\
\hline UNL1691 & 27 & 1.0 & 4.3 & 2.54 & 0.6 & 2.1 & 0.19 & $2.45 \pm 0.10$ & $24.63 \pm 0.77$ & $10.0 \pm 0.6$ & 20 & 12 \\
\hline UNL1684 & 28 & 1.0 & 3.8 & 3.05 & 0.7 & 2.8 & 0.19 & $2.98 \pm 0.11$ & $27.58 \pm 1.32$ & $9.2 \pm 0.6$ & 20 & 22 \\
\hline UNL1713 & 29 & 1.0 & 7.9 & 1.67 & 0.6 & 1.6 & 0.19 & $1.67 \pm 0.07$ & $19.73 \pm 0.44$ & $11.8 \pm 0.7$ & 26 & 10 \\
\hline UNL1709 & 30 & 1.0 & 5.8 & 1.84 & 0.7 & 2.5 & 0.19 & $1.92 \pm 0.08$ & $18.94 \pm 0.56$ & $9.8 \pm 0.6$ & 25 & 14 \\
\hline UNL1686 & 31 & 1.0 & 4.5 & 1.89 & 0.7 & 2.5 & 0.19 & $1.99 \pm 0.08$ & $20.84 \pm 0.75$ & $10.4 \pm 0.7$ & 20 & 16 \\
\hline UNL1685 & 32 & 1.0 & 2.2 & 1.95 & 0.6 & 2.4 & 0.19 & $2.06 \pm 0.08$ & $19.51 \pm 0.73$ & $9.8 \pm 0.6$ & 31 & 15 \\
\hline UNL1689 & 33 & 1.0 & 3.8 & 1.86 & 0.6 & 2.2 & 0.19 & $1.93 \pm 0.08$ & $19.23 \pm 0.99$ & $10.0 \pm 0.7$ & 20 & 20 \\
\hline UNL880 & 34 & 1.0 & 5.2 & 1.95 & 0.5 & 2.0 & 0.20 & $1.94 \pm 0.08$ & $17.70 \pm 0.53$ & $9.1 \pm 0.5$ & 46 & 18 \\
\hline UNL1419 & 35 & 1.0 & 7.8 & 1.69 & 0.5 & 1.6 & 0.19 & $1.66 \pm 0.07$ & $17.17 \pm 0.74$ & $10.3 \pm 0.7$ & 26 & 21 \\
\hline UNL890 & 36 & 1.0 & 3.2 & 2.32 & 0.6 & 2.5 & 0.19 & $2.34 \pm 0.07$ & $19.72 \pm 0.52$ & $8.4 \pm 0.5$ & 42 & 15 \\
\hline UNL1404 & 37 & 1.0 & 6.2 & 2.53 & 0.7 & 2.7 & 0.19 & $2.46 \pm 0.10$ & $22.86 \pm 0.58$ & $9.3 \pm 0.5$ & 30 & 14 \\
\hline UNL1418 & 38 & 1.0 & 6.3 & 3.25 & 1.0 & 4.3 & 0.19 & $3.18 \pm 0.13$ & $27.57 \pm 0.69$ & $8.7 \pm 0.5$ & 31 & 13 \\
\hline UNL893 & 39 & 1.0 & 5.5 & 1.66 & 0.6 & 2.7 & 0.19 & $1.79 \pm 0.07$ & $15.36 \pm 0.63$ & $8.6 \pm 0.6$ & 27 & 21 \\
\hline UNL878 & 40 & 1.0 & 6.4 & 1.67 & 0.7 & 2.3 & 0.19 & $1.78 \pm 0.07$ & $17.47 \pm 0.82$ & $9.8 \pm 0.7$ & 34 & 23 \\
\hline UNL882 & 41 & 1.0 & 6.8 & 1.93 & 0.6 & 2.1 & 0.19 & $1.93 \pm 0.08$ & $16.65 \pm 0.83$ & $8.6 \pm 0.6$ & 30 & 26 \\
\hline UNL886 & 42 & 1.0 & 4.9 & 2.10 & 0.5 & 1.9 & 0.19 & $2.07 \pm 0.08$ & $18.21 \pm 0.73$ & $8.8 \pm 0.6$ & 33 & 21 \\
\hline UNL1694 & 43 & 1.0 & 4.7 & 2.34 & 0.5 & 2.1 & 0.19 & $2.26 \pm 0.08$ & $25.23 \pm 0.32$ & $11.2 \pm 0.6$ & 21 & 6 \\
\hline UNL1695 & 44 & 1.0 & 4.4 & 2.06 & 0.4 & 1.7 & 0.19 & $2.00 \pm 0.08$ & $16.94 \pm 0.84$ & $8.4 \pm 0.6$ & 25 & 23 \\
\hline UNL1696 & 45 & 1.0 & 3.0 & 2.40 & 0.5 & 2.2 & 0.19 & $2.36 \pm 0.09$ & $23.01 \pm 0.89$ & $9.8 \pm 0.6$ & 22 & 17 \\
\hline UNL820 & 46 & 1.0 & 8.9 & 1.76 & 0.4 & 1.5 & 0.19 & $1.67 \pm 0.07$ & $14.18 \pm 0.76$ & $8.5 \pm 0.6$ & 25 & 18 \\
\hline UNL887 & 47 & 1.0 & 5.7 & 2.42 & 0.5 & 2.0 & 0.19 & $2.31 \pm 0.09$ & $19.73 \pm 0.67$ & $8.5 \pm 0.5$ & 31 & 17 \\
\hline UNL823 & 48 & 1.0 & 4.9 & 3.43 & 0.6 & 3.1 & 0.19 & $3.21 \pm 0.13$ & $22.97 \pm 0.96$ & $7.2 \pm 0.5$ & 30 & 21 \\
\hline UNL1409 & 49 & 1.0 & 7.6 & 2.93 & 0.9 & 3.6 & 0.19 & $2.83 \pm 0.11$ & $23.00 \pm 0.68$ & $8.1 \pm 0.5$ & 33 & 16 \\
\hline
\end{tabular}




\begin{tabular}{|c|c|c|c|c|c|c|c|c|c|c|c|c|}
\hline UNL822 & 50 & 1.0 & 5.8 & 2.52 & 0.4 & 1.7 & 0.19 & $2.33 \pm 0.09$ & $19.88 \pm 0.89$ & $8.5 \pm 0.6$ & 27 & 22 \\
\hline UNL824 & 51 & 1.0 & 5.0 & 2.30 & 0.4 & 1.9 & 0.19 & $2.19 \pm 0.09$ & $18.50 \pm 0.81$ & $8.4 \pm 0.6$ & 24 & 20 \\
\hline UNL828 & 52 & 1.0 & 4.8 & 1.45 & 0.3 & 1.2 & 0.19 & $1.46 \pm 0.06$ & $13.86 \pm 0.84$ & $9.5 \pm 0.8$ & 24 & 27 \\
\hline UNL884 & 53 & 1.0 & 5.1 & 2.69 & 0.6 & 2.3 & 0.19 & $2.58 \pm 0.10$ & $21.19 \pm 0.73$ & $8.2 \pm 0.5$ & 29 & 17 \\
\hline UNL827 & 54 & 1.0 & 12.4 & 1.59 & 0.3 & 1.2 & 0.19 & $1.45 \pm 0.06$ & $15.21 \pm 0.68$ & $10.5 \pm 0.7$ & 23 & 20 \\
\hline UNL826 & 55 & 1.0 & 5.0 & 1.46 & 0.5 & 2.3 & 0.19 & $1.59 \pm 0.06$ & $12.52 \pm 0.51$ & $7.9 \pm 0.5$ & 29 & 18 \\
\hline Sample & $\begin{array}{c}\text { Site } \\
\#\end{array}$ & $\begin{array}{l}\text { Depth } \\
\text { (m) }\end{array}$ & $\begin{array}{l}\mathrm{H}_{2} \mathrm{O}^{*} \\
(\mathrm{wt} \%)\end{array}$ & $\begin{array}{c}\mathrm{K}_{2} \mathrm{O} \\
(\mathrm{wt} \%)\end{array}$ & $\begin{array}{c}\mathrm{U} \\
(\mathrm{ppm})\end{array}$ & $\begin{array}{l}\text { Th } \\
\text { (ppm) }\end{array}$ & $\begin{array}{l}D_{\text {cosmic }}{ }^{\dagger} \\
(\mathrm{Gy} / \mathrm{ka})\end{array}$ & $\begin{array}{c}D_{\text {total }} \\
(\mathrm{Gy} / \mathrm{ka})\end{array}$ & $\begin{array}{c}D_{\mathrm{e}}^{\S} \\
(\mathrm{Gy}) \\
\end{array}$ & $\begin{array}{l}\mathrm{Age}^{\#} \\
(\mathrm{ka}) \\
\end{array}$ & $\begin{array}{l}\text { Aliquots** } \\
\text { (n) }\end{array}$ & $\begin{array}{l}\mathrm{OD}^{\dagger \dagger} \\
(\%)\end{array}$ \\
\hline UNL1406 & 56 & 1.0 & 1.8 & 1.77 & 0.5 & 1.7 & 0.19 & $1.85 \pm 0.07$ & $18.51 \pm 0.49$ & $10.0 \pm 0.6$ & 31 & 13 \\
\hline UNL2515 & 57 & 1.0 & 5.0 & 1.23 & 1.00 & 1.56 & 0.19 & $1.44 \pm 0.09$ & $13.10 \pm 0.80$ & $9.1 \pm 0.9$ & 21 & 25 \\
\hline UNL1413 & 58 & 1.0 & 2.8 & 1.36 & 0.5 & 1.6 & 0.19 & $1.50 \pm 0.06$ & $14.19 \pm 0.46$ & $9.5 \pm 0.6$ & 32 & 17 \\
\hline UNL2769 & 59 & 1.0 & 4.8 & 1.65 & 0.58 & 2.02 & 0.19 & $1.74 \pm 0.09$ & $17.22 \pm 0.69$ & $9.9 \pm 0.7$ & 17 & 14 \\
\hline UNL2771 & 60 & 1.0 & 2.8 & 1.68 & 0.48 & 1.75 & 0.19 & $1.76 \pm 0.08$ & $16.27 \pm 0.40$ & $9.2 \pm 0.5$ & 30 & 12 \\
\hline UNL2772 & 61 & 1.0 & 5.0 & 1.95 & 0.88 & 2.88 & 0.19 & $2.09 \pm 0.11$ & $20.05 \pm 0.74$ & $9.6 \pm 0.6$ & 20 & 14 \\
\hline UNL2770 & 62 & 2.0 & 3.2 & 2.51 & 0.77 & 2.42 & 0.17 & $2.50 \pm 0.11$ & $24.50 \pm 0.68$ & $9.8 \pm 0.5$ & 26 & 13 \\
\hline UNL2516 & 63 & 1.0 & 5.0 & 3.33 & 1.45 & 2.93 & 0.19 & $3.21 \pm 0.24$ & $23.20 \pm 1.50$ & $7.2 \pm 0.8$ & 20 & 19 \\
\hline
\end{tabular}

Eolian silt cap ages

\begin{tabular}{|c|c|c|c|c|c|c|c|c|c|c|c|c|}
\hline Sample & $\begin{array}{c}\text { Site } \\
\#\end{array}$ & $\begin{array}{c}\text { Depth } \\
(\mathrm{m})\end{array}$ & $\begin{array}{l}\mathrm{H}_{2} \mathrm{O}^{*} \\
(\mathrm{wt} \%)\end{array}$ & $\begin{array}{c}\mathrm{K}_{2} \mathrm{O} \\
(\mathrm{wt} \%) \\
\end{array}$ & $\begin{array}{c}\mathrm{U} \\
(\mathrm{ppm})\end{array}$ & $\begin{array}{c}\text { Th } \\
\text { (ppm) }\end{array}$ & $\begin{array}{c}D_{\text {cosmic }}{ }^{\dagger} \\
\text { (Gy/ka) }\end{array}$ & $\begin{array}{c}D_{\text {total }} \\
(\mathrm{Gy} / \mathrm{ka})\end{array}$ & $\begin{array}{c}D_{\mathrm{e}}^{\S} \\
(\mathrm{Gy}) \\
\end{array}$ & $\begin{array}{c}\mathrm{Age}^{\#} \\
(\mathrm{ka})\end{array}$ & $\begin{array}{l}\text { Aliquots** } \\
\text { (n) }\end{array}$ & $\begin{array}{l}\mathrm{OD}^{\dagger 1} \\
(\%)\end{array}$ \\
\hline UNL1073 & 11 & 0.10 & 24.5 & 3.04 & 1.5 & 4.5 & 0.21 & $2.64 \pm 0.14$ & $23.16 \pm 0.46$ & $8.8 \pm 0.6$ & 40 & 12 \\
\hline UNL1074 & 12 & 0.20 & 16.7 & 2.42 & 1.1 & 3.3 & 0.21 & $2.28 \pm 0.11$ & $25.13 \pm 0.74$ & $11.1 \pm 0.7$ & 36 & 17 \\
\hline UNL1072 & 13 & 0.22 & 21.8 & 2.60 & 1.3 & 4.1 & 0.21 & $2.37 \pm 0.12$ & $21.85 \pm 0.36$ & $9.3 \pm 0.6$ & 42 & 10 \\
\hline UNL1077 & 23 & 0.25 & 26.2 & 2.89 & 1.7 & 5.0 & 0.21 & $2.58 \pm 0.14$ & $25.21 \pm 0.59$ & $9.8 \pm 0.7$ & 40 & 15 \\
\hline UNL1075 & 24 & 0.30 & 16.8 & 2.47 & 1.4 & 4.3 & 0.21 & $2.42 \pm 0.11$ & $25.68 \pm 0.87$ & $10.5 \pm 0.6$ & 37 & 20 \\
\hline UNL1076 & 25 & 0.23 & 33.7 & 2.52 & 1.7 & 4.9 & 0.21 & $2.19 \pm 0.14$ & $27.61 \pm 0.87$ & $12.7 \pm 1.0$ & 30 & 18 \\
\hline UNL1711 & 64 & 1.0 & 10.6 & 1.51 & 0.6 & 2.5 & 0.19 & $1.56 \pm 0.06$ & $17.93 \pm 0.77$ & $11.5 \pm 0.8$ & 20 & 18 \\
\hline UNL1712 & 65 & 1.0 & 13.7 & 1.59 & 1.2 & 3.9 & 0.19 & $1.77 \pm 0.08$ & $24.48 \pm 0.95$ & $13.8 \pm 0.9$ & 22 & 17 \\
\hline
\end{tabular}

* In-situ water content; assumption of $30 \%$ error

${ }^{\dagger}$ Calculated using Prescott and Hutton (1994)

$\S_{ \pm 1}$ standard error; calculated using central age model (CAM) of Galbraith et al. (1999); 5-mm disks ( 1000 grains) used for all samples

\# \pm 1 standard deviation; years before 2007

** Accepted aliquots

†' Overdispersion (Galbraith, 2005) 
Table DR2. Sample lab numbers, site numbers, names, locations, and elevations

\begin{tabular}{|c|c|c|c|c|c|}
\hline Lab \# (UNL) & Site \# & Site Name & Latitude $\left({ }^{\circ} \mathrm{N}\right)$ & Longitude $\left({ }^{\circ} \mathrm{W}\right)$ & Elevation (m asl) \\
\hline 1212 & 1 & No Name Rd & $46^{\circ} 28.54^{\prime}$ & $86^{\circ} 5.90^{\prime}$ & 265 \\
\hline 1208 & 2 & Mid Driggs & $46^{\circ} 24.70^{\prime}$ & $86^{\circ} 8.66^{\prime}$ & 250 \\
\hline 1213 & 3 & E Branch Fox: M77 & $46^{\circ} 23.72^{\prime}$ & $85^{\circ} 57.02^{\prime}$ & 231 \\
\hline 1207 & 4 & Pines & $46^{\circ} 21.20^{\prime}$ & $86^{\circ} 16.84^{\prime}$ & 245 \\
\hline 1211 & 5 & Ecklund & $46^{\circ} 21.80^{\prime}$ & $86^{\circ} 10.34^{\prime}$ & 246 \\
\hline 881 & 6 & Seney RR & $46^{\circ} 20.71^{\prime}$ & $86^{\circ} 8.77^{\prime}$ & 235 \\
\hline 892 & 7 & Seney Sand Pit & $46^{\circ} 20.29^{\prime}$ & $85^{\circ} 56.25^{\prime}$ & 230 \\
\hline 1688 & 8 & Hardwood Isle N & $46^{\circ} 20.38^{\prime}$ & $85^{\circ} 52.59^{\prime}$ & 222 \\
\hline 821 & 9 & Wannamaker & $46^{\circ} 21.31^{\prime}$ & $85^{\circ} 47.46^{\prime}$ & 224 \\
\hline 1411 & 10 & E Branch Fox: M28 & $46^{\circ} 20.05^{\prime}$ & $85^{\circ} 49.05^{\prime}$ & 217 \\
\hline 1073 & 11 & Blue Mountains 1-5 & $46^{\circ} 18.44^{\prime}$ & $85^{\circ} 52.11^{\prime}$ & 236 \\
\hline 1074 & 12 & Blue Mountains 1-8 & $46^{\circ} 18.49^{\prime}$ & $85^{\circ} 53.06^{\prime}$ & 235 \\
\hline 1072 & 13 & Blue Mountains 1-1 & $46^{\circ} 18.62^{\prime}$ & $85^{\circ} 53.05^{\prime}$ & 235 \\
\hline 2514 & 14 & Blue Mountains & $46^{\circ} 17.71^{\prime}$ & $85^{\circ} 55.52^{\prime}$ & 219 \\
\hline 879 & 15 & Refuge Edge & $46^{\circ} 12.78^{\prime}$ & $85^{\circ} 58.27^{\prime}$ & 206 \\
\hline 2510 & 16 & Fletcher Hill 2 & $46^{\circ} 9.19^{\prime}$ & $86^{\circ} 19.07^{\prime}$ & 226 \\
\hline 894 & 17 & Mint Farm & $46^{\circ} 8.66^{\prime}$ & $86^{\circ} 21.33^{\prime}$ & 210 \\
\hline 2509 & 18 & Fletcher Hill 1 & $46^{\circ} 10.87^{\prime}$ & $86^{\circ} 19.98^{\prime}$ & 223 \\
\hline 883 & 19 & Stutz & $46^{\circ} 9.52^{\prime}$ & $86^{\circ} 16.58^{\prime}$ & 210 \\
\hline 2511 & 20 & Rollaway Dune 1 & $46^{\circ} 3.84^{\prime}$ & $86^{\circ} 13.75^{\prime}$ & 216 \\
\hline 2512 & 21 & Rollaway Dune 2 & $46^{\circ} 5.51^{\prime}$ & $86^{\circ} 15.32^{\prime}$ & 222 \\
\hline 895 & 22 & Bear Creek & $46^{\circ} 4.75^{\prime}$ & $86^{\circ} 3.36^{\prime}$ & 198 \\
\hline 1077 & 23 & Niagara 2-15 & $46^{\circ} 3.76^{\prime}$ & $86^{\circ} 3.84^{\prime}$ & 237 \\
\hline 1075 & 24 & Niagara 2-14 & $46^{\circ} 3.36^{\prime}$ & $86^{\circ} 4.08^{\prime}$ & 226 \\
\hline 1076 & 25 & Niagara 2-11 & $46^{\circ} 2.68^{\prime}$ & $86^{\circ} 3.19^{\prime}$ & 206 \\
\hline 1692 & 26 & Low Rollaway & $46^{\circ} 4.04^{\prime}$ & $86^{\circ} 15.58^{\prime}$ & 204 \\
\hline 1691 & 27 & Sturgeon Hole & $46^{\circ} 1.95^{\prime}$ & $86^{\circ} 15.34^{\prime}$ & 193 \\
\hline 1684 & 28 & Niagara Quarry & $46^{\circ} 1.79^{\prime}$ & $86^{\circ} 15.75^{\prime}$ & 192 \\
\hline 1713 & 29 & Gillingham & $46^{\circ} 1.35^{\prime}$ & $86^{\circ} 10.95^{\prime}$ & 204 \\
\hline 1709 & 30 & Judy Graves & $46^{\circ} 1.28^{\prime}$ & $86^{\circ} 10.94^{\prime}$ & 217 \\
\hline 1686 & 31 & Esky Paper Mid & $46^{\circ} 0.87^{\prime}$ & $86^{\circ} 11.67^{\prime}$ & 191 \\
\hline 1685 & 32 & Esky Paper Top & $46^{\circ} 0.75^{\prime}$ & $86^{\circ} 11.70^{\prime}$ & 195 \\
\hline 1689 & 33 & Bosanic & $46^{\circ} 1.18^{\prime}$ & $86^{\circ} 10.99^{\prime}$ & 217 \\
\hline 880 & 34 & RRTT & $46^{\circ} 12.46^{\prime}$ & $86^{\circ} 50.31^{\prime}$ & 248 \\
\hline 1419 & 35 & Shoe Pac & $46^{\circ} 1.13^{\prime}$ & $86^{\circ} 30.72^{\prime}$ & 225 \\
\hline 890 & 36 & Iron Creek & $46^{\circ} 0.79^{\prime}$ & $86^{\circ} 28.44^{\prime}$ & 220 \\
\hline 1404 & 37 & Ramsey Lake & $45^{\circ} 58.65^{\prime}$ & $86^{\circ} 45.44^{\prime}$ & 228 \\
\hline 1418 & 38 & Ogonz & $45^{\circ} 56.48^{\prime}$ & $86^{\circ} 47.46^{\prime}$ & 222 \\
\hline 893 & 39 & 10 Mile Rapids & $45^{\circ} 56.68^{\prime}$ & $86^{\circ} 41.98^{\prime}$ & 203 \\
\hline 878 & 40 & Cooks & $45^{\circ} 57.39^{\prime}$ & $86^{\circ} 28.78^{\prime}$ & 229 \\
\hline 882 & 41 & Ensign & $45^{\circ} 54.58^{\prime}$ & $86^{\circ} 50.44^{\prime}$ & 215 \\
\hline 886 & 42 & Valentine & $45^{\circ} 49.73^{\prime}$ & $86^{\circ} 29.19^{\prime}$ & 207 \\
\hline 1694 & 43 & Krause Rd & $46^{\circ} 14.54^{\prime}$ & $85^{\circ} 33.07^{\prime}$ & 227 \\
\hline 1695 & 44 & Millecoquins & $46^{\circ} 14.17^{\prime}$ & $85^{\circ} 31.79^{\prime}$ & 221 \\
\hline 1696 & 45 & Legend Land & $46^{\circ} 12.94^{\prime}$ & $85^{\circ} 33.06^{\prime}$ & 212 \\
\hline 820 & 46 & Siphon Creek & $46^{\circ} 23.39^{\prime}$ & $85^{\circ} 44.21^{\prime}$ & 232 \\
\hline
\end{tabular}




\begin{tabular}{|c|c|c|c|c|c|}
\hline 887 & 47 & Luce 442 & $46^{\circ} 23.89^{\prime}$ & $85^{\circ} 43.68^{\prime}$ & 237 \\
\hline 823 & 48 & Dollarville & $46^{\circ} 22.77^{\prime}$ & $85^{\circ} 35.05^{\prime}$ & 234 \\
\hline 1409 & 49 & NBY 2 & $46^{\circ} 21.67^{\prime}$ & $85^{\circ} 27.95$ & 225 \\
\hline 822 & 50 & Hendrix & $46^{\circ} 14.27^{\prime}$ & $85^{\circ} 19.47^{\prime}$ & 259 \\
\hline 824 & 51 & Fiborn & $46^{\circ} 14.09^{\prime}$ & $85^{\circ} 8.48^{\prime}$ & 249 \\
\hline 828 & 52 & Hendrie & $46^{\circ} 16.48^{\prime}$ & $85^{\circ} 8.13^{\prime}$ & 233 \\
\hline Lab \# (UNL) & Site \# & Site Name & Latitude $\left({ }^{\circ} \mathrm{N}\right)$ & Longitude $\left({ }^{\circ} \mathrm{W}\right)$ & Elevation (m asl) \\
\hline 884 & 53 & Trout Lake & $46^{\circ} 11.82^{\prime}$ & $85^{\circ} 1.27^{\prime}$ & 264 \\
\hline 827 & 54 & Eckerman 1 & $46^{\circ} 21.06^{\prime}$ & $85^{\circ} 0.49^{\prime}$ & 265 \\
\hline 826 & 55 & Soo Strongs & $46^{\circ} 21.53^{\prime}$ & $84^{\circ} 53.67^{\prime}$ & 282 \\
\hline 1406 & 56 & Eckerman 2 & $46^{\circ} 25.04^{\prime}$ & $85^{\circ} 4.26^{\prime}$ & 251 \\
\hline 2515 & 57 & Ken Chucky Camp & $46^{\circ} 27.63^{\prime}$ & $85^{\circ} 14.35^{\prime}$ & 229 \\
\hline 1413 & 58 & Sand Cut & $46^{\circ} 28.06^{\prime}$ & $85^{\circ} 14.41^{\prime}$ & 236 \\
\hline 2769 & 59 & GM Airport & $46^{\circ} 36.53$ & $85^{\circ} 55.62^{\prime}$ & 255 \\
\hline 2771 & 60 & Porter Creek & $46^{\circ} 35.35^{\prime}$ & $85^{\circ} 52.64^{\prime}$ & 244 \\
\hline 2772 & 61 & Barfield Lakes & $46^{\circ} 35.57^{\prime}$ & $85^{\circ} 51.39^{\prime}$ & 252 \\
\hline 2770 & 62 & Below the Rim & $46^{\circ} 33.13^{\prime}$ & $85^{\circ} 46.58^{\prime}$ & 260 \\
\hline 2516 & 63 & Jinny Palms & $46^{\circ} 44.26^{\prime}$ & $85^{\circ} 11.87^{\prime}$ & 227 \\
\hline 1711 & 64 & Pence & $46^{\circ} 32.21^{\prime}$ & $85^{\circ} 12.04^{\prime}$ & 287 \\
\hline 1712 & 65 & Amasa & $46^{\circ} 32.44^{\prime}$ & $85^{\circ} 12.50^{\prime}$ & 292 \\
\hline
\end{tabular}




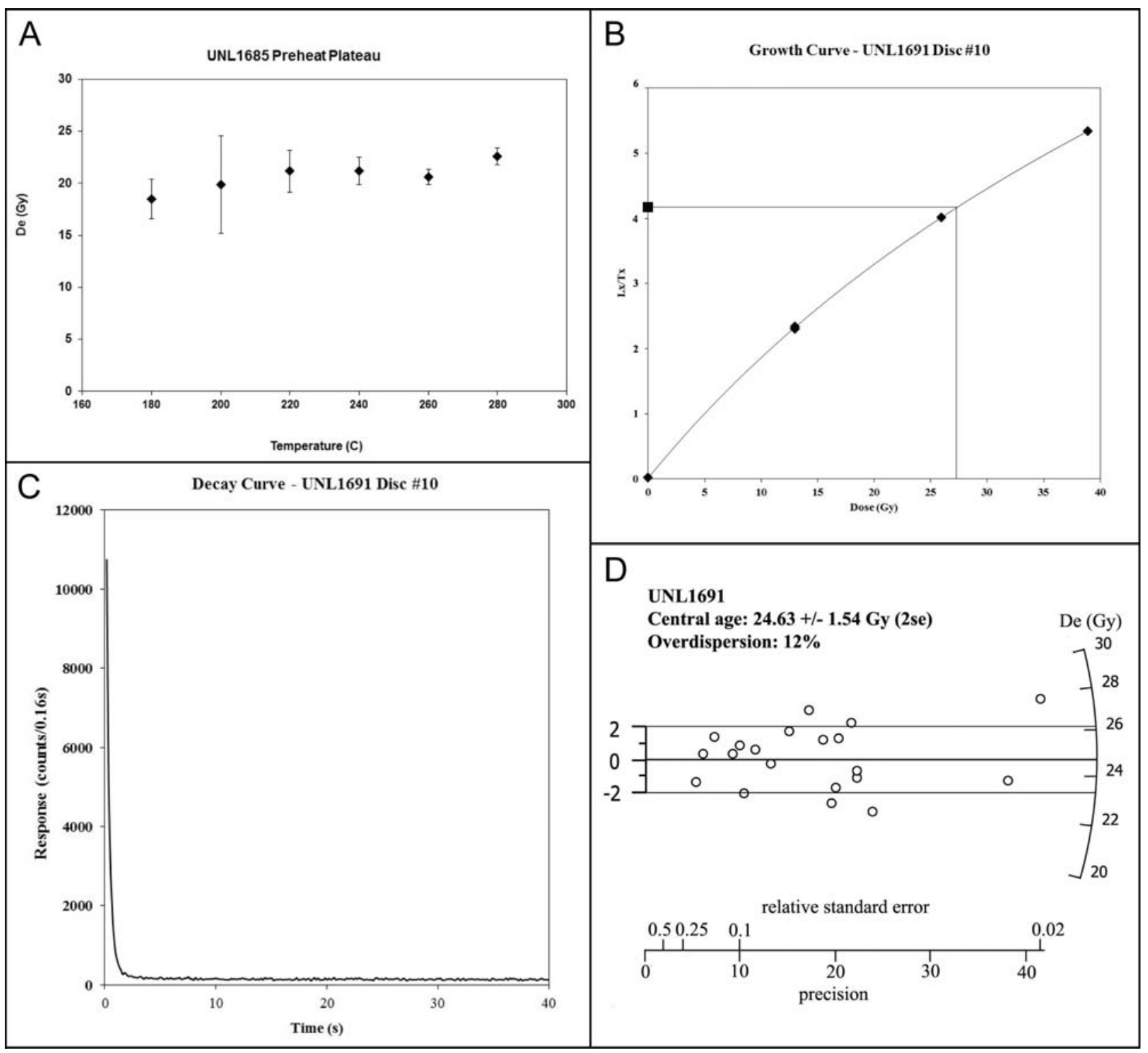

Figure DR1. Example OSL data: A) preheat plateau test, B) growth curve, C) decay curve, D) radial plot 


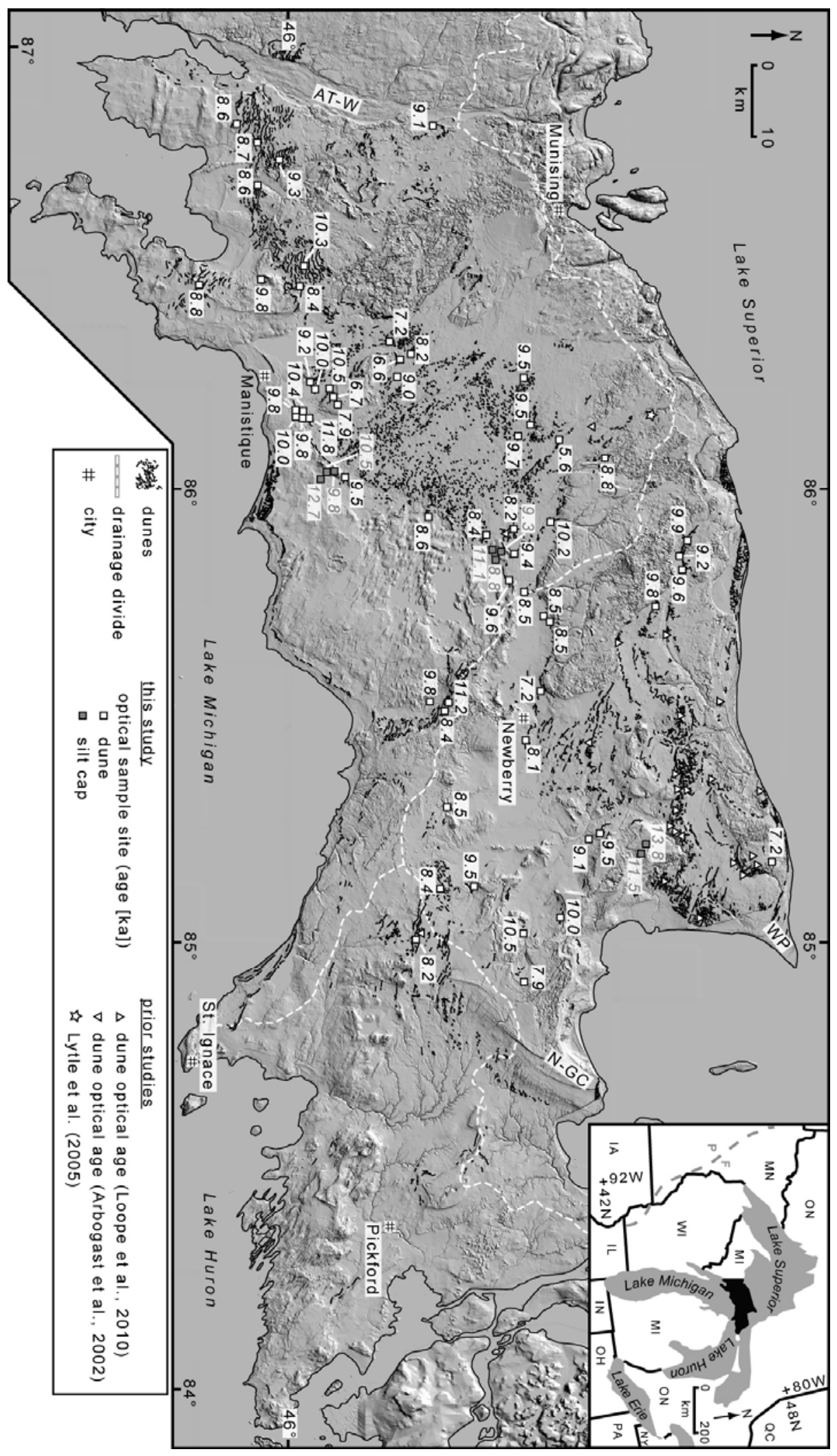

Figure DR2. 10-m hillshade digital elevation model of eastern Upper Michigan with mapped dunes and ages (Table DR1). 


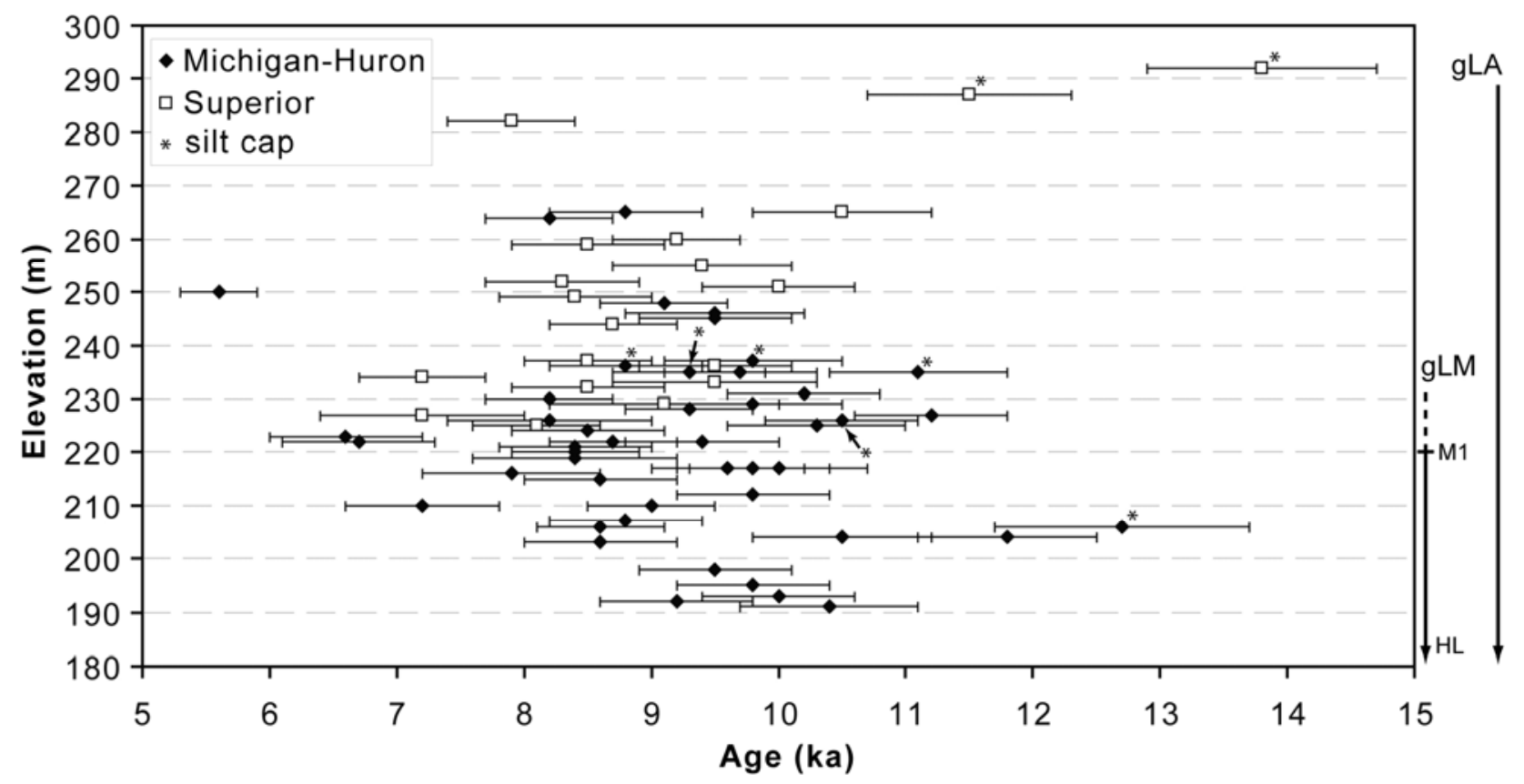

Figure DR3. Elevation vs. age plot of dune and silt cap samples divided by drainage basin (Michigan-Huron and Superior). Error on ages is $\pm 1 \sigma$. Note the lack of relationship between elevation and dune age, suggesting climatic and vegetation control on sand availability and dune building. Vertical lines at right end are elevational ranges of glacial Lake Minong (gLM) and glacial Lake Algonquin (gLA). Dashed line denotes a $12 \mathrm{~m}$ transgression (Breckenridge et al., 2010) from a main Minong strandline (horizontal bar; M1 [ 220 m] of Loope et al., 2010). Solid line denotes lake-level fall of $\sim 45 \mathrm{~m}$ from M1 to the Houghton Low (HL) at $\sim 9.3 \mathrm{ka}$ due to downcutting of the Nadoway-Gros Cap barrier (Yu et al., 2010). Lake Minong only would have influenced sites in the Superior basin (squares) and most samples from this study within the Superior basin lie above the former extent of Lake Minong. Glacial Lake Algonquin (Futyma, 1981; Schaetzl et al., 2002) occupied much of eastern Upper Michigan in both the Superior and Michigan-Huron basins from $\sim 13.0$ to $11.5 \mathrm{k}$ cal yr BP during several lake stages. Differential glacial isostatic rebound has resulted in Lake Algonquin shorelines found as high as $290 \mathrm{~m}$ in the northern part of the study area southeast of Whitefish Point and $220 \mathrm{~m}$ in the extreme southwestern part of the study area on the Garden Peninsula. 


\section{References}

Adamiec, G., and Aitken, M., 1998, Dose-rate conversion factors: update: Ancient TL, v. 16, p. 37-50.

Aitken, M.J., 1976, Thermoluminescent age evaluation and assessment of error limits: revised system: Archaeometry, v. 18, p. 233-238.

Aitken, M.J., 1985, Thermoluminescence Dating, Academic Press, 359 p.

Aitken, M.J., 1998, An Introduction to Optical Dating, Oxford University Press, 267 p.

Aitken, M.J., and Alldred, J.C., 1972, The assessment of error limits in thermoluminescent dating: Archaeometry, v. 14, p. 257-267.

Arbogast, A.F., Wintle, A.G., and Packman, S.C., 2002, Widespread middle Holocene dune formation in the eastern Upper Peninsula of Michigan and the relationship to climate and outletcontrolled lake level: Geology, v. 30, p. 55-58, doi: 10.1130/0091-7613(2002).

Bailey, R.M., Singarayer, J.S., Ward, S., and Stokes, S., 2003, Identification of partial resetting using $D_{\mathrm{e}}$ as a function of illumination time: Radiation Measurements, v. 37, p. 511-518, doi: $10.1016 / \mathrm{S} 1350-4487(03) 00063-5$.

Bailey, R.M., and Arnold, L.J., 2006, Statistical modeling of single grain quartz $\mathrm{D}_{\mathrm{e}}$ : distribution and an assessment for estimating burial dose: Quaternary Science Reviews, v. 25, p. 2475-2502, doi: 10.1016/j.quascirev.2005.09.012.

Breckenridge, A., Lowell, T.V., Fisher, T.G., and Yu, S., 2010, A late Lake Minong transgression in the Lake Superior basin as documented by sediments from Fenton Lake, Ontario: Journal of Paleolimnology, doi: 10.1007/s10933-010-9447-z.

Brennan, B.J., 2003, Beta doses to spherical grains: Radiation Measurements, v. 37, p. 299-303, doi: 10.1016/S1350-4487(03)00063-5.

Colls, A.E., Stokes, S., Blum, M.D., and Straffin, E., 2001, Age limits on the Late Quaternary evolution of the upper Loire River: Quaternary Science Reviews, v. 20, p. 743-750, doi: 10.1016/S0277-3791(00)00048-2.

Futyma, R.P., 1981, The northern limits of glacial Lake Algonquin in Upper Michigan: Quaternary Research, v. 15, p. 291-310, doi: 10.1016/0033-5894(81)90032-6.

Galbraith, R.F., 1988, Graphical display of estimates having different standard errors:

Technometrics, v. 30, p. 271-281.

Galbraith, R.F., 2005, Statistics for fission track analysis: Chapman \& Hall/CRC, 240 p. 
Galbraith, R.F., 2010, On plotting OSL equivalent doses: Ancient TL, v. 28, p. 1-9.

Galbraith, R.F., Roberts, R.G., Laslett, G.M., Yoshida, H., and Olley, J.M., 1999, Optical dating of single and multiple grains of quartz from Jinmium rock shelter, northern Australia: Part I, experimental design and statistical models: Archaeometry, v. 41, p. 339-364, doi: 10.1111/j.14754754.1999.tb00987.x.

Lavi, N., Groppi, F., and Alfassi, Z.B., 2004, On the measurement of ${ }^{40} \mathrm{~K}$ in natural and synthetic materials by the method of high-resolution gamma-ray spectrometry: Radiation Measurements, $\mathrm{v}$. 38, p. 139-143, doi: 10.1016/j.radmeas.2003.11.005.

Loope, H.M., Loope, W.L., Goble, R.J., Fisher, T.G., Jol, H.M., and Seong, J.C., 2010, Early Holocene dune activity linked with final destruction of Glacial Lake Minong, eastern Upper Michigan, USA: Quaternary Research, v. 74, p. 73-81, doi: 10.1016/j.yqres.2010.03.006.

Murray, A.S., and Olley, J.M., 2002, Precision and accuracy in the optically stimulated luminescence dating of sedimentary quartz: A status review: Geochronometria, v. 21, p. 1-16.

Murray, A.S., and Wintle, A.G., 2003, The single aliquot regenerative dose protocol: Potential for improvements in reliability: Radiation Measurements, v. 37, p. 377-381, doi: 10.1016/S13504487(03)00053-2.

Olley, J.M., Caitcheon, G.G., and Murray, A.S., 1998, The distribution of apparent dose as determined by optically stimulated luminescence in small aliquots of fluvial quartz: Implications for dating young samples: Quaternary Science Reviews, v. 17, p. 1033-1040, doi: 10.1016/S02773791(97)00090-5.

Olley, J.M., Caitcheon, G., and Roberts, R.G., 1999, The origin of dose distributions in fluvial sediments, and the prospect of dating single grains from fluvial deposits using optically stimulated luminescence: Radiation Measurements, v. 30, p. 207-217, doi: 10.1016/S1350-4487(99)00040-2.

Prescott, J.R., and Hutton, J.T., 1994, Cosmic ray contributions to dose rates for luminescence and ESR dating: large depths and long-term time variations: Radiation Measurements, v. 23, p. 497500, doi: 10.1016/1350-4487(94)90086-8.

Preusser, F., and Kasper, H.U., 2001, Comparison of dose rate determination using high-resolution gamma spectrometry and inductively coupled plasma - mass spectrometry: Ancient TL, v. 19, p. $19-23$.

Schaetzl, R.J., Drzyzga, S.A., Weisenborn, B.N., Kincare, K.A., Lepczyk, X.C., Shein, K., Dowd, C.M., and Linker, J., 2002, Measurement, correlation, and mapping of glacial Lake Algonquin shorelines in Northern Michigan: Annals of the Association of American Geographers, v. 92, p. 399-415, doi: 10.1111/1467-8306.00296. 
Schaetzl, R.J., and Loope, W.L., 2008, Evidence for an eolian origin for the silt-enriched soil mantles on the glaciated uplands of eastern Upper Michigan, USA: Geomorphology, v. 100, p. 285-295, doi: 10.1016/j.geomorph.2008.01.002.

Wintle, A.G., and Murray, A.S., 2006, A review of quartz optically stimulated luminescence characteristics and their relevance in single-aliquot regeneration dating protocols: Radiation Measurements, v. 41, p. 369-391, doi: 10.1016/j.radmeas.2005.11.001.

Yu, S.-Y., Colman, S.M., Lowell, T.V., Milne, G.A., Fisher, T.G., Breckenridge, A., Boyd, M., and Teller, J.T., 2010, Freshwater outburst from Lake Superior as a trigger for the cold event 9300 years ago: Science, v. 328, p. 1262-1266, doi: 10.1126/science.1187860. 